\title{
Benefits of multimodal therapy for patients with osteoarthritis
}

\author{
Mihaela-Ana Nicolau', Ruxandralonescu²,3 \\ ${ }^{1} \mathrm{CMI}$ Dr. Nicolau Mihaela-Ana, Pitesti, Romania \\ "Sf. Maria" Hospital, Bucharest, Romania \\ 3"Carol Davila" University of Medicine and Pharmacy, Bucharest, Romania
}

\begin{abstract}
Objectives. The purpose of this study is to underline the benefits of the complex, multimodal therapy, composed of the pharmacological and non-pharmacological treatment of patients suffering from osteoarthritis (OA) with different localizations, namely pain and functioning relief and the increase of the quality of life.

Methods. The study included 246 patients with osteoarthritis diagnosed at the clinical examination and confirmed by radiological investigations. All the patients were pharmacologically treated and 127 of them also followed non-pharmacological treatment consisting of a recuperation program kinesiotherapy and electrotherapy.

Results. Patients who achieved a significant decrease in pain were those treated with multimodal therapy (pharmacologically, kinesiotherapy and electrotherapy) $27.3 \%$, followed by patients treated only pharmacologically $20 \%$, and patients treated pharmacologically plus kinesiotherapy (without electrotherapy) $10.7 \%$. Patients who achieved moderate improvement in 2 out of 3 (pain, function / mobility, global assessment), according to OARSI also responded to treatment, that means $85.7 \%$ who combined pharmacological treatment and kinesiotherapy (without electrotherapy) and only $67 \%$ subjects who received only pharmacological treatment.

Conclusions. Our findings demonstrate that multimodal therapy, which consists of the combination of pharmacological and non-pharmacological treatment, brings important benefits to the management of patients with OA.

Keywords: osteoarthritis, pharmacological treatment, non-pharmacological treatment,
\end{abstract} multimodal therapy, kinesiotherapy

\section{INTRODUCTION}

OA affects approximately $10 \%$ of the global population and represents the second cause of invalidity among people over 50 years old (1).

The direct social-economical costs of OA can be very high (2), showing the impact that this affection has over the society (public health systems, patients) and the necessity to find new therapeutical strategies to help reduce the pain, the functional improvement and the increase of the quality of life for affected people.

Recent research has put a special emphasis on understanding the mechanisms of pain ("Personalized analgesia") (3), considering that subjects with OA show diffuse hyperalgesia to mechanical stimuli or heat (4).

Patients with hip and knee OA who are overweight should be encouraged to lose weight and maintain their weight at a lower level. They express pain, the determinant symptom of their disability (5), discomfort, fatigue, stiffness and sleep disorders (6). In time, these can cause vicious attitudes, muscle weakness and walking disorders (7).

The therapeutical approach in OA is based on the recommendations of the American College of Rheumatology (ACR) from 2012, the Osteoarthritis Research Society International (OARSI) from 2008 and 2014 and those of the European League Against Rheumatism (EULAR) (8), which suggest that the optimal management of patients with OA involves a personalized combination of pharmacological and non-pharmacological treatment, taking into account the value system of the patient, along with its preferences $(9,10)$, because it seems that only a therapeutical method alone cannot offer a proper clinical response. 
This combined, multimodal therapy is at its beginning and still requires multiple appropriate clinical researches. According to the research conducted so far, this therapy has beneficial but moderate effects, and the patient has to continue exercise at home for its effects to last in time. Programs include therapeutical exercises, hydrotherapy (11), but also manual therapy such as muscle stretches, mobilization exercises and maintenance of soft tissues (12).

The evidence-based OARSI 2014 recommendations point out that non-pharmacological exercise consisting of physical exercise is indicated for all patients with knee OA.

\section{OBJECTIVES}

The purpose of this study is to underline the benefits of the complex, multimodal therapy, composed of pharmacological and non-pharmacological treatment of patients suffering from OA with different localizations, more precisely the pain relief, the functioning and the increase of the quality of life.

\section{MATERIAL AND METHODS}

In this prospective unicentric study, 246 patients were evaluated during the 2013-2017 period with the following criteria of inclusion: the OA diagnosis based on ACR criteria, age between 18-87 years old and the agreement of patients to take part in the study.

The exclusion criteria were: patients found in an evolutionary stage of the disease, patients with cardiac insufficiency, myocardial infarction or coronarian bypass -3 months before the study, patients suffering from hypertension with values hard to control, chronic obstructive bronchopneumopathy, cachexia, tuberculosis, febrile states, severe mental affections, pregnant or breastfeeding women, alcohol addicts and those with surgical indication (knee or hip replacement).

Patients with spondylarthrosis, with surgical indication based on magnetic resonance imaging (MRI) were excluded, but the patients who had been operated were included.

OA diagnosis was suggested by the clinical exam and confirmed by paraclinical and radiological investigations.

The pharmacological treatment was followed by all patients, provided that the treatment remained the same throughout the study period.

All the patients who wanted to undertake kinesiotherapy were treated twice a week, for a period of approximately 5 weeks (10 sessions). Patients were treated individually. Exercise therapy was tailored to the patient's individual needs.

As data collection tools, structured questionnaires were used: the index functional Lequesne and the WOMAC index (on a scale of 0 to 4 divided into 5 items) for the evaluation of knee and hip OA and the global assessment HAQ questionnaire for all types of osteoarthritis that evaluates activities of daily living (ADL) and the quality of life of patients with OA.

Functional tests Lequesne take into consideration: pain assessment, maximum walking distance, impairment of daily activities. The three areas are summed up in a severity index. The analysis compares either the total severity index or the indices calculated separately on the three areas.

Three scales for pain (VAS, Likert, a new pain scale) and a range-type mobility scale were used to evaluate the research variables.

The OA were classified according to their radiographic degree: early to moderate, advanced and severe OA.

The recovery program consisted of manual therapy and exercises or just exercise therapy. Each session lasted 45 minutes and had the role of increasing muscle strength, coordination, maintaining the amplitude of movement, reducing pain and increasing the quality of life.

Manual therapy consisted of stretching or shortened muscles and manipulations of the spine and lower limbs.

Exercise therapy was given according to the protocol van Baar. It included exercises for strength and length, coordination and mobility and exercises for locomotion abilities. Also, instructions for the home exercises were given. Exercise therapy in OA aims at relief of pain and reduction of disability.

Furthermore, some patients followed electrotherapy (Transcutaneous Electro Stimulation - TENS) to control pain and muscle relaxation, except for patients with joint replacement hip or knee. For the latter, electrotherapy is contraindicated.

The IBM SPSS 25.0 for Mac Book was used to process the statistical data. Descriptive statistics and comparisons between different patient groups, depending on the type of osteoarthritis, have been made. Comparisons were made between the result type variables. 


\section{RESULTS}

All the patients received pharmacological treatment and a part of these were treated non-pharmacologically.

Non-pharmacological treatment consisted of manual therapy (performed by the physiotherapist) combined with exercises or just exercise therapy.

We conducted a mixed analysis on treatment types and relative change criteria (Fig. 1) between visit 1 (before treatment) and visit 2 (after treatment).

Non-pharmacologically treated patients - manual therapy and exercises - obtained the most significant value for relative pain change $65.5 \%$.

Subjects treated with exercise therapy only gained the highest value for the relative pain change $68.9 \%$ and small values were recorded for the relative change Lequesne distance $32.7 \%$.

According to the type of therapeutic intervention, the patients treated pharmacologically and with kinesiotherapy (without electrotherapy) obtained an increased value for the relative pain change $69.6 \%$ and a reduced value for the relative change of the distance Lequesne $25.0 \%$.

Subjects treated only pharmacologically experienced a significant Relative VAS Change and a reduced value for relative change Lequesne pain $27.4 \%$.

Pharmacologically treated, with kinesiotherapy and electrotherapy achieved a high value for relative pain change $65.3 \%$ and a reduce value for relative Lequesne pain change $32.0 \%$.

We further analyzed the response to treatment using the OARSI criteria $(13,14)$, depending on the therapy followed.

We believe that patients who have experienced a significant decrease in pain have responded to the treatment.
Patients who achieved moderate improvement in 2 out of 3 (pain, function/mobility, global assessment), according to OARSI $(9,13)$, also responded to treatment.

According to the analysis, the following did not get treatment response: $13.4 \%$ of patients without kinesiotherapy, $5.2 \%$ of patients who have performed manual therapy and exercises and $10 \%$ of those who did only exercise therapy.

The following achieved a significant decrease in pain: $19.3 \%$ of patients without kinesiotherapy, $28.9 \%$ of subjects who received manual therapy and exercises and $6.7 \%$ of those who had only exercise therapy.

The following showed a moderate improvement of 2 out of 3 (pain, function/mobility, global assessment) according to OARSI: $67.2 \%$ of patients without kinesiotherapy, $66 \%$ of those who benefited from manual therapy and exercises and $83.3 \%$ exercise therapy (Fig. 2).

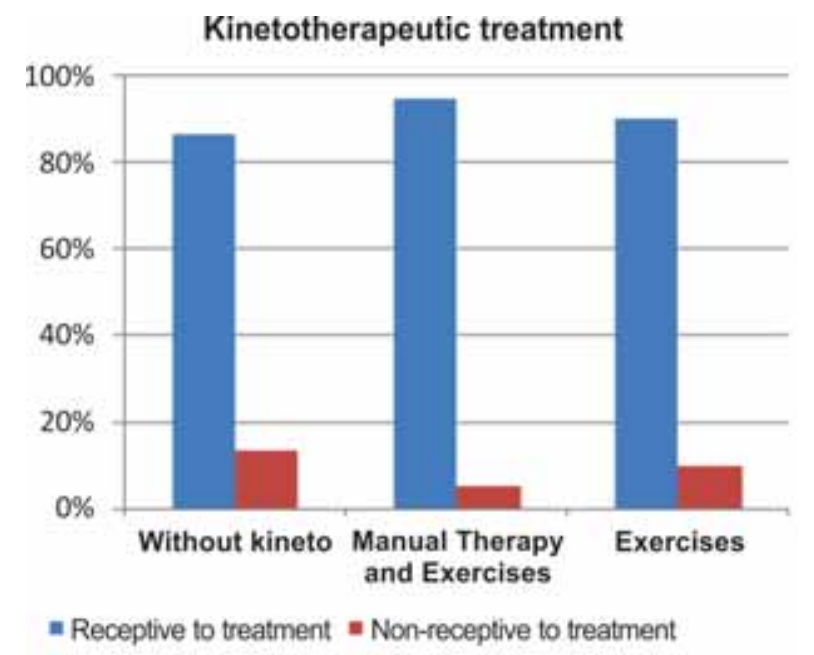

FIGURE 2. Representation of treatment response analysis using OARSI A - according to: Treatment

After the type of therapeutic intervention, patients who achieved a significant decrease in pain were:

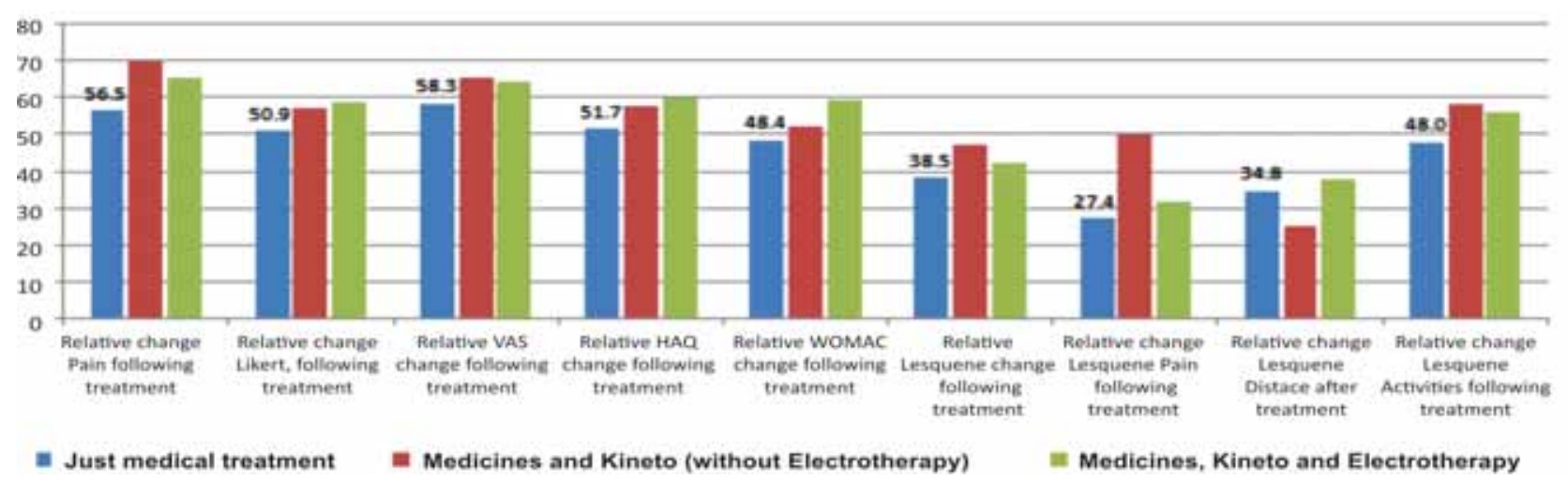

FIGURE 1. Representation of mixed analysis of types of treatment 
- patients treated pharmacologically, kinesiotherapy and electrotherapy $-27.3 \%$

- patients only pharmacologically treated $20 \%$

- patients treated pharmacologically plus kinesiotherapy (without electrotherapy) 10.7\%.

After the type of therapeutic intervention, subjects who achieved a moderate improvement of 2 out of 3 (pain, function/mobility, global assessment) were pharmacologically treated patients who also practiced kinesiotherapy (without electrotherapy) $85.7 \%$, subjects treated only pharmacologically $67 \%$ and patients treated pharmacologically, plus kinesiotherapy and electrotherapy (Fig. 3).

\section{DISCUSSIONS}

Regarding the response to treatment based on the therapy followed, we noticed that the highest efficacy of the treatment achieved was when the patients combined pharmacological and non-pharmacological treatment, respectively manual therapy and exercises.

Also, for the subjects in the sample, manual therapy and exercises led to the strongest reduction in pain, but exercise therapy mostly contributed to a moderate improvement in 2 out of 3 pain, function / mobility, global evaluation.

Depending on the kinesiotherapeutic treatment followed, patients who received manual therapy and exercises obtained the highest treatment response rate while subjects who did not have kinesiotherapy had the highest non-response to treatment.

Regarding to the type of therapeutic intervention, the highest efficiency of the treatment was achieved by patients who combined pharmacological and kinesiotherapy (without electrotherapy), and the high-

Type of therapeutic intervention

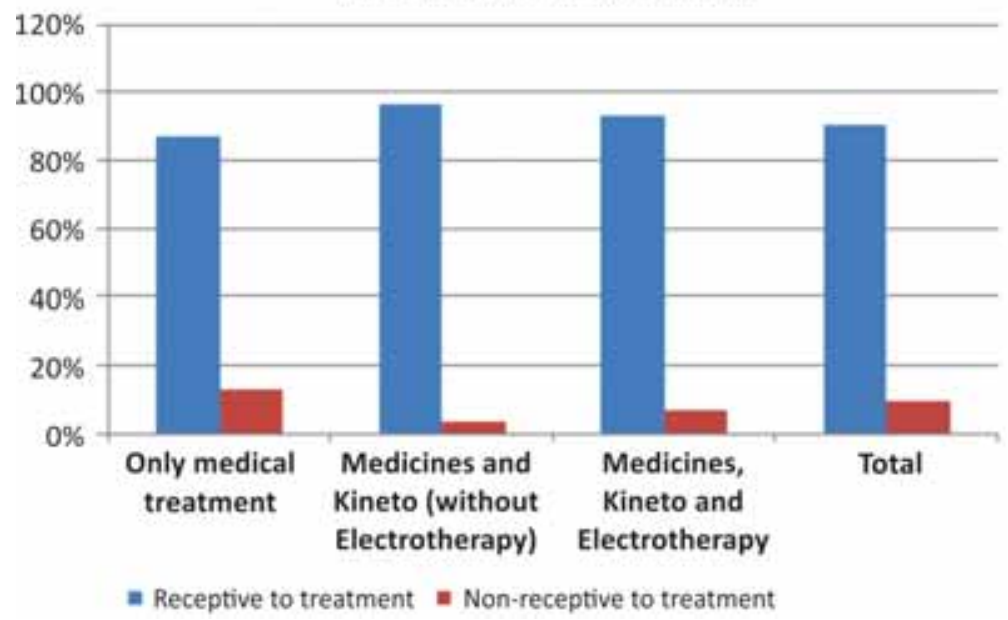

est non-response to treatment were the subjects who received only pharmacological treatment.

As a drawback of the study, we can say that patients have not been monitored for a longer time (1 year), and global evaluation can be criticized for its sensitivity.

OARSI recommends further validation in additional data sets in other pharmacological and non-pharmacological clinical trials.

\section{CONCLUSIONS}

The present study emphasizes the importance of combining the two types of pharmacological and non-pharmacological treatment for optimal management of patients with $\mathrm{OA}$, their pain relief, functional improvement and quality of life.

The most beneficial outcome was found in OA patients doing muscles strengthening and range of motion exercises or stretching muscles and manipulation of the joints.

Obviously, beneficial effects post-treatment were found for pain and for disability. Manual therapy and exercises or exercise therapy reduces pain and disability in patients with OA, especially for hip and knee OA.

However, the size of the effects is medium for pain and global assessment and small for the other effects.

However, we did not notice a suggestive change after treatment with regard to the Lequesne Distance Index $(34.5 \%)$ for patients with hip or knee OA.

This shows that after following the treatment, at the opposite pole stand the patients who could not achieve the performance of walking longer distances without feeling the same pain as they felt before the treatment.

This was probably due to the well-known sedentarism of subjects with hip or knee OA.
FIGURE 3. Representation of treatment response analysis using OARSI A - according to: The type of therapeutic intervention 


\section{REFERENCES}

1. Helmick C.G., Felson D.T., Lawrence R.C. et al. Estimates of prevalence of arthritis and other rheumatic conditions in the United States. Arthritis Rheum 2008; 58: 15-25

2. Rabenda V., Manette C., Lemmens R. et al. Direct and indirect costs attributable to osteoarthritis in active subjects. J Rheumatol 2006; 33(6): 1152-1158

3. Mease P.J., Hanna S., Frakes E.P. et al. Pain mechanisms in osteoarthritis: understanding the role of central pain and current approaches to its treatment. J Rheumatol 2011; 38: 1546-51

4. Phillips K., Claw D.J. Central pain mechanisms in rheumatic diseases. Future directions. Arthritis Rheum 2013; 65 (2): 291-302

5. Van Baar M.E., Dekker J., Lemmens J.A. et al. Pain and disability in patients with osteoarthritis of hip or knee: the relationship with articular, kinesiological and psychological characteristics. Journal of Rheumatology 1998; 25: 125-33

6. Gooberman-Hill R., Woolhead G., MacKichan F. et al. Assessing chronic joint pain: lessons from a focus group study. Arthritis Care Res 2007; 57:667-71.

7. Lane N.E. Clinical practice. Osteoarthritis of the hip. N Engl J Med 2007; 357:1413-1421

8. Hochberg M.C., Altman R.D., April K. et al. American College of Rheumatology 2012 recommendations for the use of nonpharmacologic and pharmacologic therapies in osteoarthritis of hand, hip and knee. Arthritis Care Res 2012; 64:465-74.

9. Hugo L., Hoeksma D., Cornelia H.M. et al. A comparison of the OARSI response criteria with patient's global assessment in patients with osteoarthritis of the hip treated with a nonpharmacological intervention. OsteoArthritis and Cartilage 2006; 14:77-81.

10. Cutolo M., Berenbaum F., Hochberg, M. et al. Commentary on recent therapeutic guidelines for osteoarthritis. Seminars in Arthritis and Rheumatism 2015; volume 44(6): 611-617.

11. Silva L.E., Valim V., Pessanha A.P. et al. Hydrotherapy versus conventional land-based exercise for the management of patients with osteoarthritis of the knee: a randomized clinical trial. Phys Ther 2008; 88(1):12-21

12. Deyle G.D., Allison S.C., Matekel R.L. et al. Physical therapy treatment effectiveness for osteoarthritis of the knee: a randomized comparison of supervised clinical exercise and manual therapy procedures versus a home exercise program. Phys Ther 2005; 85:1301-17.

13. Dougados M., LeClaire P., van der Heijde et al. A report of the Osteoarthritis Research Society International Standing Comittee for Clinical Trials Response Criteria Initiative. Osteoarthritis and Cartilage 2000; 8: 395-403.

14. Altman R., Brandt K., Hochberg M. et al. Design and conduct of clinical trials of patients with osteoarthritis. Recommendations from a task force of the Osteoarthritis research Society. Osteoarthritis and Cartilage 1996; 4: 226-227.

Conflict of interest: none declared Financial support: none declared 